\title{
Composición, diversidad y estructura del ensamble de plantas leñosas en dos paleocauces con diferente intervención antrópica del Chaco semiárido, Argentina
}

\author{
Composition, diversity, and structure of woody vegetation in two ancient watercourses with \\ different human interventions in the semiarid Chaco of Argentina
}

\author{
Silvana Cardozo ${ }^{\text {a*}}$, Andrés Tálamo ${ }^{\mathrm{b}}$, Federico Mohr ${ }^{\mathrm{c}}$ \\ * Autor de correspondencia: ${ }^{a}$ Universidad Nacional de Salta, Facultad de Ciencias Naturales, Cátedra de Botánica Agrícola, \\ Salta, Argentina, tel.: 0387-154185220. silca_cardozo@hotmail.com

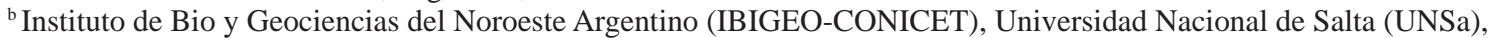 \\ Salta, Argentina. \\ ${ }^{\text {c } U n i v e r s i d a d ~ N a c i o n a l ~ d e ~ S a l t a, ~ F a c u l t a d ~ d e ~ C i e n c i a s ~ N a t u r a l e s, ~ S a l t a, ~ A r g e n t i n a . ~}$
}

\begin{abstract}
SUMMARY
For many decades the semiarid Chaco ecosystems of Argentina have experienced anthropogenic disturbances, such as fire and cattle grazing, that have shaped the present-day vegetation in concert with climatic and edaphic conditions. For example, the dynamic relationship between grassland and woodland may be altered by the frequency and intensity of fire and cattle grazing. We analyzed woody vegetation among sectors of two ancient watercourses with similar geomorphological histories; one undisturbed for some time (Copo National Park) and the other with a long history of cattle grazing and fire (Copo Provincial Reserve). The undisturbed site had lower total species richness, diversity, basal area, density of both adults and seedlings, and cover of woody vegetation than the disturbed site, and doubled the number of branches per adult shrub or tree. Species composition of woody plants was notably different between the two sites. Considering the similar geomorphological history of the two ancient watercourses, we concluded that much of the contrast in their woody vegetation is likely due to land use history, especially their very different fire regimes.
\end{abstract}

Key words: shrub encroachment, grassland-woodland dynamics, disturbance, livestock, shrub invasion, fire.

\section{RESUMEN}

Los ecosistemas del Chaco argentino vienen siendo perturbados por la actividad humana desde hace décadas, especialmente por la ganadería extensiva y el fuego, que actuando con distinta frecuencia e intensidad modelan el paisaje junto al clima y la fisiografía. Los pastizales del Chaco son colonizados por plantas leñosas y las perturbaciones mencionadas podrían modificar la sucesión secundaria en dichos ambientes. El objetivo fue comparar el ensamble de plantas leñosas y la cobertura del suelo entre sectores de un paleocauce sin perturbaciones antrópicas (paleocauce no intervenido, Parque Nacional Copo) y sectores de un paleocauce similar con perturbaciones antrópicas: ganado y fuego (pastizal intervenido, Reserva Provincial Copo). En el paleocauce intervenido se registraron menores valores de área basal, densidad total (adultas y renovales) y cobertura del suelo, y se duplicó el número de ramas promedio comparado con el paleocauce no intervenido. La composición de especies de plantas leñosas fue significativamente diferente entre los paleocauces. Se concluye que los paleocauces estudiados resultaron muy diferentes en prácticamente todas las variables comparadas y, dada su similar geomorfología, es posible que gran parte de lo encontrado esté explicado por las diferencias en las perturbaciones antrópicas de ambos pastizales, principalmente por el régimen de fuego del pastizal intervenido.

Palabras clave: arbustos, colonización, perturbaciones, ganado, fuego.

\section{INTRODUCCIÓN}

La colonización de pastizales naturales por plantas leñosas nativas es un proceso ampliamente conocido en distintos ambientes del mundo (Van Auken 2000, 2009). Entre las causas potenciales que promueven dicha colonización suelen mencionarse al cambio global, elevados niveles de $\mathrm{CO}_{2}$ en la atmósfera, cambios en las presiones de competencia, cambios en la frecuencia del fuego, altos niveles de herbivoría (que disminuiría la acumulación del material fino combustible y reduciría la frecuencia del fue- go), cambios en las tasas de dispersión de semillas por el ganado y otros animales silvestres, y la posible interacción entre dos o varios de estos factores (Van Auken 2009). Sin embargo, las causas y las consecuencias del proceso de colonización no están del todo clarificadas y son temas de actuales debates (Maestre et al. 2009). Debido a la complejidad de las respuestas y dependiendo de la forma de vida y de la región estudiada los resultados son muy variables. A modo de ejemplo, se ha propuesto que la colonización de plantas leñosas en pastizales es la principal expresión de un proceso de desertificación de pastizales 
áridos y semiáridos (Millennium Ecosystem Assessment 2005), mientras que, por otro lado, Maestre et al. (2009) postulan que la colonización de arbustos puede ser un paso importante en revertir el proceso de desertificación en pastizales mediterráneos.

En la ecorregión del Gran Chaco, que comprende un mosaico de diferentes ambientes (Prado 1993), las sabanas y los pastizales también constituyen escenarios en donde ocurre la colonización de plantas leñosas, proceso que puede ser modificado por factores como el régimen de fuego y la ganadería (Morello 1970, Morello y Amámoli 1968, Cabral et al. 2003). En el Gran Chaco, el fuego ha sido históricamente utilizado por los aborígenes chaqueños ya sea para cazar o con fines bélicos y actualmente es utilizado para el control de plantas leñosas y para promover el rebrote de pastos y así aumentar la oferta forrajera (Bravo et al. 2001, Kunst et al. 2003). Por lo tanto, en estos ambientes ambas perturbaciones ocurren generalmente de manera simultánea y es muy poco lo que se conoce de sus posibles efectos sobre el proceso de colonización de plantas leñosas en los pastizales del Chaco semiárido que crecen sobre paleocauces. El régimen del fuego es un factor importante para explicar el proceso de colonización de arbustos sobre pastizales, ya que si se reduce la frecuencia o intensidad de los incendios de pastizales, los arbustos tienen más posibilidades de reclutamiento y de expansión de individuos existentes en el pastizal; si a su vez se disminuye la presión de pastoreo, el combustible fino vegetal puede incrementar considerablemente contribuyendo a aumentar la competencia de los pastos con los arbustos, y a potenciar los efectos ya mencionados del régimen de fuego recurrente de pastizales (Cabral et al. 2003, Briggs et al. 2005, Van Auken 2009). En este mismo sentido, se ha propuesto al sobrepastoreo como la causa de la inminente desaparición de pastizales "puros" (con dominio de gramíneas) del Chaco (Morello y Saravia Toledo 1959b, Neumann 1999) y el consecuente avance de las plantas leñosas sobre los pastizales.

Por todo esto, se postula que la colonización de pastizales del Chaco semiárido por el ensamble de plantas leñosas puede depender, entre otros factores, de la intensidad y frecuencia del uso ganadero y del fuego. Para acercarse a este objetivo general, en esta aproximación no manipulativa comparamos dos paleocauces con diferente historia de perturbaciones: un paleocauce sometido a las perturbaciones tradicionales (fuego periódico y pastoreo de ganado vacuno, llamado de ahora en adelante paleocauce intervenido) y otro paleocauce sin historia de incendios ni de pastoreo (llamado paleocauce no intervenido). Si las perturbaciones estudiadas no modificaron el proceso de colonización, se espera que el estado actual del ensamble de leñosas no varíe entre ambos paleocauces comparados. Por lo tanto, el objetivo específico es comparar la biodiversidad de plantas leñosas, densidad, número de ramas y el área basal de dichas plantas leñosas y la cobertura del suelo entre sectores del paleocauce no intervenido y sectores del paleocauce intervenido.

\section{MÉTODOS}

La subregión de los antiguos cauces del río Juramento del Chaco semiárido es una paleollanura surcada por numerosos cauces arenosos de dirección noroeste-sudeste denominados paleocauces, ríos muertos o "caños". Sobre estas geoformas se desarrollan los pastizales naturales del Chaco semiárido, dominados por aibe (Elionurus muticus Spreng.) y por lo tanto son sectores utilizados por los pobladores locales por su alta abundancia de forraje para el ganado vacuno (Morello y Saravia Toledo 1959b, Morello y Adámoli 1968).

El clima es marcadamente estacional, con el $80 \%$ de las precipitaciones anuales $(700 \mathrm{~mm})$ concentradas entre octubre y marzo (primavera-verano) y la vegetación dominante en superficie que se encuentra en los interfluvios (zonas ubicadas entre paleocauces) es un bosque de quebracho blanco (Aspidosperma quebracho-blanco Schtdl.), quebracho colorado santiagueño (Schinopsis lorentzii (Griseb.) Engl.) y mistol (Zyziphus mistol Griseb.)(Tálamo y Caziani 2003, Tálamo 2006).

El estudio se realizó en el Área de Conservación Copo, Santiago del Estero, Argentina (figura 1). Dentro del Parque Nacional Copo se seleccionó un paleocauce sin intervención antrópica conocida (ni ganado ni fuego recurrente evidente, Perovic et al. 2008) y dentro de la Reserva Provincial Copo se seleccionó un paleocauce sometido al uso tradicional que hacen los habitantes locales (puesteros criollos): pastoreo por ganadería vacuna extensiva y fuego programado pero sin control, con un régimen de fuego del cual sólo se pudo caracterizar su alta frecuencia (generalmente cada dos años) (llamado de ahora en adelante "paleocauce intervenido").

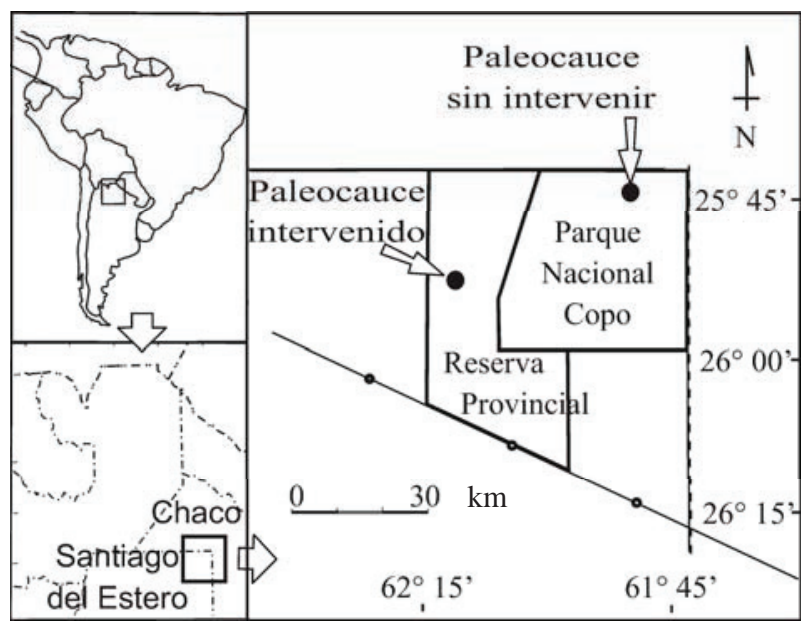

Figura 1. Ubicación del Área de Conservación Copo (Parque Nacional y Reserva Provincial), Santiago del Estero (Argentina) y de los dos paleocauces con diferente intervención antrópica. Location of Copo Conservation Unit (National Park and Provincial Reserve), Santiago del Estero (Argentina), indicating the two ancient watercourses with different disturbance histories. 
Diseño del estudio. El factor de diseño utilizado fue "paleocauces" y presentó dos niveles discretos: paleocauce no intervenido y paleocauce intervenido. En cada paleocauce seleccionado se estudió el ensamble de plantas leñosas usando parcelas rectangulares (10 parcelas en cada pastizal distanciadas por no menos de 200 m entre sí, que fueron consideradas las unidades experimentales para los análisis estadísticos). Cada parcela consistió en un rectángulo de $2 \mathrm{~m} \times 100 \mathrm{~m}$ donde se registraron las especies de todos los individuos leñosos con diámetro mayor a $0,5 \mathrm{~cm}$ medido a una altura de $20 \mathrm{~cm}$ del suelo. Los individuos con un diámetro menor a $0,5 \mathrm{~cm}$ se consideraron renovales y se estimó su densidad. La cobertura de suelo se registró sobre 51 estimaciones puntuales (distanciados por 1 metro) distribuidos sobre la línea central de la parcela. Las categorías de cobertura consideradas fueron: hojarasca (hojas secas), gramíneas, latifoliadas, madera (corteza o ramas), pteridofitas y suelo desnudo (sin cobertura). Las variables de respuestas analizadas fueron: a) riqueza específica (número de especies), b) diversidad y composición de especies, c) área basal total y por especie, d) densidad total y por especie, e) número promedio de ramas/individuo y f) cobertura del suelo en las categorías mencionadas.

Análisis de datos. Se comparó la riqueza específica mediante curvas de rarefacción, técnica que permite estimar la riqueza de especies esperada a un mismo nivel de abundancia; las curvas se construyeron usando el programa EcoSim (Gotelli y Entsminger 2002), el cual calcula la riqueza promedio de muestras aleatorias de abundancia creciente de individuos, con 1.000 repeticiones para cada nivel de abundancia. La diversidad de especies se analizó mediante curvas de rango-abundancia las cuales permiten comparar gráficamente la riqueza de especies (número de puntos), sus abundancias relativas, la forma de las curvas (equitatividad) y la secuencia de cada una de las especies que componen la comunidad sin perder su identidad (Feinsinger 2004). Para evaluar si ambos paleocauces se diferencian en cuanto a su composición de especies, se utilizó una técnica multivariante conocida como procedimiento de permutación de respuesta múltiple (MRPP), que permite saber si las diferencias en composición de especies encontradas entre los grupos comparados son mayores que las esperadas por el azar (McCune et al. 2002); dicho análisis se realizó mediante el programa estadístico PC-ORD (McCune y Mefford 1999). El área basal, la densidad de individuos y el número medio de ramas por individuo, se analizaron mediante la prueba no paramétrica de MannWhitney (Zar 1999). Al trabajar con este tipo de pruebas estadísticas, y dada la asimetría pronunciada de las variables estudiadas, las medidas de tendencia central y de dispersión utilizadas fueron la mediana y el rango intercuartil, respectivamente. El nivel de significación original fue de 0,05 , pero debido a que se realizaron múltiples pruebas estadísticas sobre un mismo conjunto de réplicas, dicho valor se ajustó mediante el procedimiento de Bonferro- ni (nivel de significación $/ \mathrm{n}^{\circ}$ de pruebas realizadas). Para saber si las distintas categorías de cobertura del suelo se distribuyeron de manera similar entre ambos pastizales se utilizó la prueba $\chi^{2}$ (Chi cuadrado) de homogeneidad (Zar 1999).

\section{RESULTADOS}

Se registró un total de 21 especies de plantas leñosas en el paleocauce no intervenido y 16 especies en el paleocauce intervenido. Comparando los dos sitios a un mismo nivel de abundancia, ambos pastizales presentaron una riqueza de especies similar ya que la riqueza máxima del paleocauce intervenido (16 especies) quedó comprendida dentro del intervalo de confianza del $95 \%$ de la riqueza promedio del paleocauce no intervenido al mismo nivel de abundancia (figura 2).

La diversidad específica de plantas leñosas en ambos pastizales fue diferente. El paleocauce no intervenido presentó mayor riqueza y fue menos equitativo que el paleocauce intervenido. En el paleocauce no intervenido dominó la especie Aloysia scorodonioides (Kunth) Cham (palo ángel), seguida por Celtis pallida Torr (tala) y Schinus poligamus (Cav.) Cabrera (molle). Mientras que el paleocauce intervenido estuvo dominado por Gochnatia argentina Cabrera (cabrera), seguida por $A$. scorodonioides y Acacia aroma Gillies ex Hook. et Arn. (tusca) (figura 3, cuadro 1). La composición de especies fue significativamente diferente entre los paleocauces comparados ya que la probabilidad de que la diferencia observada provenga de

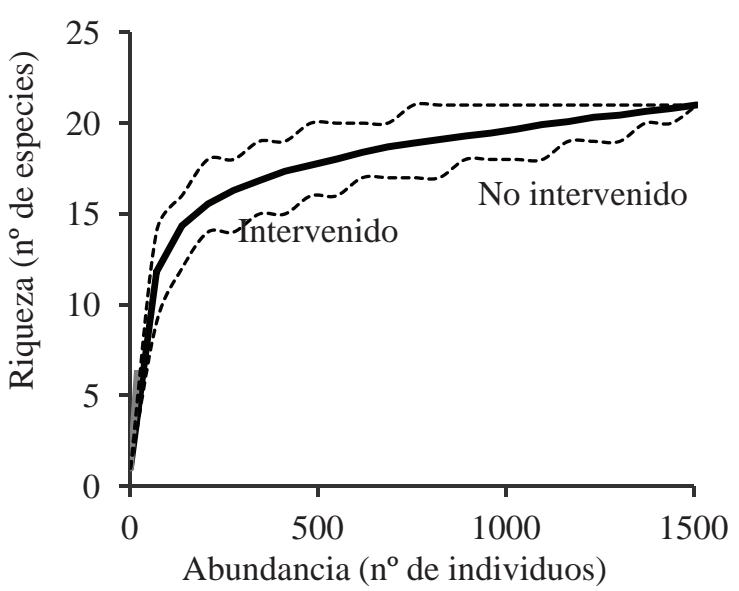

Figura 2. Curvas de rarefacción para ambos paleocauces con diferente intervención antrópica. Unidad de Conservación Copo, Santiago del Estero, Argentina. Las líneas de puntos delimitan la banda de confianza del $95 \%$ para la riqueza promedio del paleocauce no intervenido.

Rarefaction curves of species richness of woody plants for the two ancient watercourses with different human interventions, Copo Conservation Unit, Santiago del Estero, Argentina. Dotted lines delimit the $95 \%$ confidence interval for average species richness of the undisturbed ancient watercourses. 


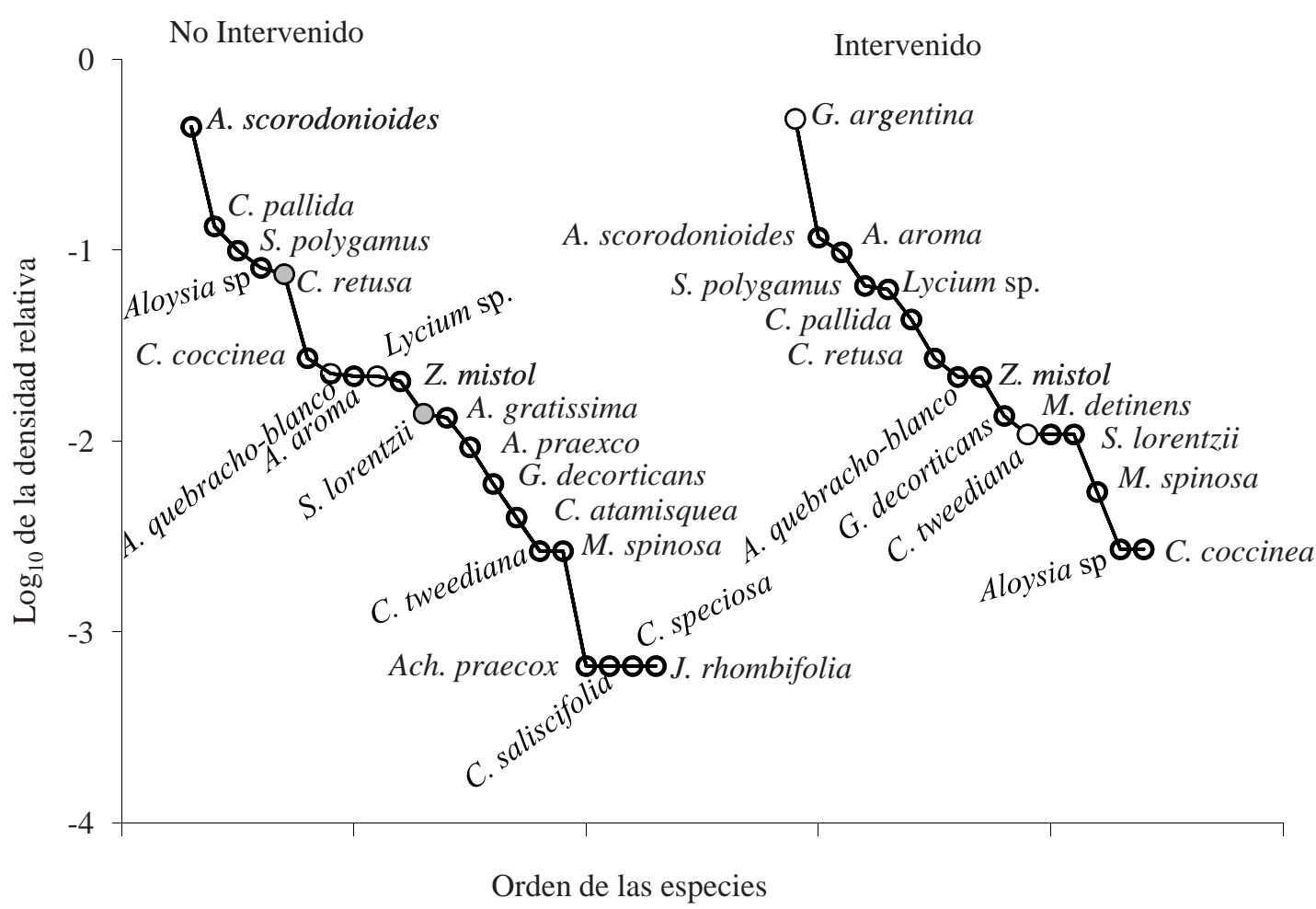

Figura 3. Curvas de rango-abundancia para ambos paleocauces con diferente intervención antrópica. Unidad de Conservación Copo, Santiago del Estero, Argentina.

Rank-abundance curves for the two ancient watercourses with different human interventions. Copo Conservation Unit, Santiago del Estero, Argentina.

paleocauces con igual composición fue muy baja (MRPP: $\mathrm{T}=-9,52$; delta observado $=50,76 ; P<0,0001)$.

El área basal total resultó diferente entre los paleocauces $\left(\mathrm{U}=155 ; \mathrm{n}_{\text {interv. }}=\mathrm{n}_{\text {nointerv. }}=10 ; P<0,0002\right.$; figura $\left.4 \mathrm{~A}\right)$ destacándose el elevado valor de área basal del paleocauce no intervenido que superó en 24 veces el área basal del paleocauce intervenido. En la mayoría de las parcelas del paleocauce no intervenido, las especies que tuvieron mayor área basal fueron S. polygamus, A. scorodonioides y A. aroma, mientras que en el paleocauce intervenido fueron A. aroma, S. polygamus y A. scorodonioides.

El paleocauce no intervenido fue el sitio con mayor densidad de plantas leñosas (cuadro 1), superando seis veces la densidad encontrada en el paleocauce intervenido $\left(\mathrm{U}=149 ; \mathrm{n}_{\text {interv. }}=\mathrm{n}_{\text {nointerv. }}=10 ; P<0,0009\right.$; figura 4B). Las especies dominantes fueron $A$. scorodonioides representando el $38 \%$ de la densidad total, C. pallida (12\%) y $S$. polygamus (11\%) en el paleocauce no intervenido, mientras que A. aroma (17\% de la abundancia total), S. polygamus (12\%) y A. scorodonioides (10\%) dominaron la densidad en el paleocauce intervenido (figura 3).

En cuanto a la forma de crecimiento de los arbustos, se encontró que el número de ramas promedio por individuo registrado en el paleocauce intervenido superó 1,62 veces el número de ramas promedio registrado en el paleocauce no intervenido ( $\mathrm{U}=65 ; \mathrm{n}_{\text {interv. }}=\mathrm{n}_{\text {nointerv. }}=10 ; P<0,0025$; figura 4C). Las especies de plantas leñosas con mayor número de ramas en el paleocauce intervenido fueron: A. aroma (llegando a cuantificar un individuo con 78 ramas), G. argentina y S. polygamus, mientras que en el paleocauce no intervenido fueron $A$. scorodonioides, C. pallida y S. polygamus.

La cobertura de suelo registrada en los puntos de ambos pastizales resultó diferente $\left(\chi^{2}=92,59 ; P<0,0001\right.$; figura 5). En el paleocauce no intervenido se registró la presencia de madera (no así en el paleocauce intervenido) y el suelo presentó una mayor cobertura de latifoliadas y hojarasca, y una menor proporción de gramíneas y suelo desnudo en comparación al paleocauce intervenido.

\section{DISCUSIÓN}

Los dos paleocauces estudiados (intervenido y no intervenido) resultaron diferentes en casi todas las variables comparadas: diversidad y composición de especies, área basal, densidad de individuos (adultos y renovales), número de ramas por individuo y cobertura del suelo. $\mathrm{Si}$ bien los paleocauces tienen una historia de intervención antrópica muy diferente (sin intervención conocida vs. con usos tradicionales: ganado y fuego recurrente), no se pueden asociar ciertamente las diferencias encontradas con su historia de uso o con la presencia o ausencia de perturba- 
Cuadro 1. Densidad total de individuos (individuos $\mathrm{x} 100 \mathrm{~m}^{-2}$ ) de todas las especies de plantas leñosas registradas en ambos paleocauces con diferente intervención antrópica. Unidad de Conservación Copo, Santiago del Estero, Argentina. Se muestran la mediana y entre paréntesis el rango intercuartil.

Total density (individuals $100 \mathrm{~m}^{-2}$ ) of all woody plants recorded in two ancient watercourses with different human interventions in Copo Conservation Unit, Santiago del Estero, Argentina. The values shown are the median and (brackets) the interquartile range.

\begin{tabular}{|c|c|c|c|}
\hline Especies (ordenadas alfabéticamente) & Familia & $\begin{array}{c}\text { Paleocauce } \\
\text { no intervenido }\end{array}$ & $\begin{array}{l}\text { Paleocauce } \\
\text { intervenido }\end{array}$ \\
\hline Acacia aroma Gillies ex Hook. et Arn. & Fabaceae & $2,5(2)$ & $3,5(2,50)$ \\
\hline Acacia praecox Griseb. & Fabaceae & $0 *$ & 0 \\
\hline Achatocarpus praecox Griseb. & Achatocarpaceae & $0,5(2)$ & 0 \\
\hline Aloysia sp. & Verbenaceae & $11,5(10,75)$ & $0 *$ \\
\hline Aloysia gratísima (Gillies et Hook. ex Hook.) Tronc. & Verbenaceae & $0(0,75)$ & 0 \\
\hline Aloysia scorodonioides (Kunth) Cham. & Verbenaceae & $49,5(49,25)$ & $2(4,50)$ \\
\hline Aspidosperma quebracho-blanco Schtdl. & Apocynaceae & $2,5(3,75)$ & $1(1)$ \\
\hline Capparis retusa Griseb. & Capparaceae & $4(11)$ & $0,5(1)$ \\
\hline Capparis atamisquea Kuntze & Capparaceae & $0 *$ & 0 \\
\hline Capparis saliscifolia Griseb. & Capparaceae & $0 *$ & 0 \\
\hline Capparis speciosa Griseb. & Capparaceae & $0 *$ & 0 \\
\hline Capparis tweediana Eichler & Capparaceae & $0 *$ & $0 *$ \\
\hline Castela coccínea Griseb. & Simaroubaceae & $3(3,50)$ & $0 *$ \\
\hline Celtis pallida Torr. & Celtidaceae & $16(13,50)$ & $1,5(2,75)$ \\
\hline Geoffroea decorticans (Gillies ex Hook. et Arn.) Burkart & Fabaceae & 0 & $0 *$ \\
\hline Gochnatia argentina (Cabrera) Cabrera & Asteraceae & $0 *$ & $0(12)$ \\
\hline Jodina rhombifolia (Hook. et Arn.) Reissek & Santalaceae & $0 *$ & 0 \\
\hline Lycium sp. & Solanaceae & $0(2)$ & $0,5(3,75)$ \\
\hline Maytenus spinosa (Griseb.) Lourteig et O’Donell & Celastraceae & $0(1)$ & $0 *$ \\
\hline Mimosa detinens Benth. & Fabaceae & 0 & $0 *$ \\
\hline Schinopsis lorentzii (Griseb.) Engl. & Anacardiaceae & $1(1,75)$ & $0(0,75)$ \\
\hline Schinus poligamus (Cav.) Cabrera & Anacardiaceae & $15(10,50)$ & $2,5(3,50)$ \\
\hline Zizyphus mistol (Grises) & Rhamnaceae & $3,5(2)$ & $0(1)$ \\
\hline Total & & $130,5(61,25)$ & $20,5(18)$ \\
\hline
\end{tabular}

*: Especies que, a pesar de presentar 0 como valor de la Mediana y del Rango Intercuartil, estuvieron presentes en una o dos parcelas.
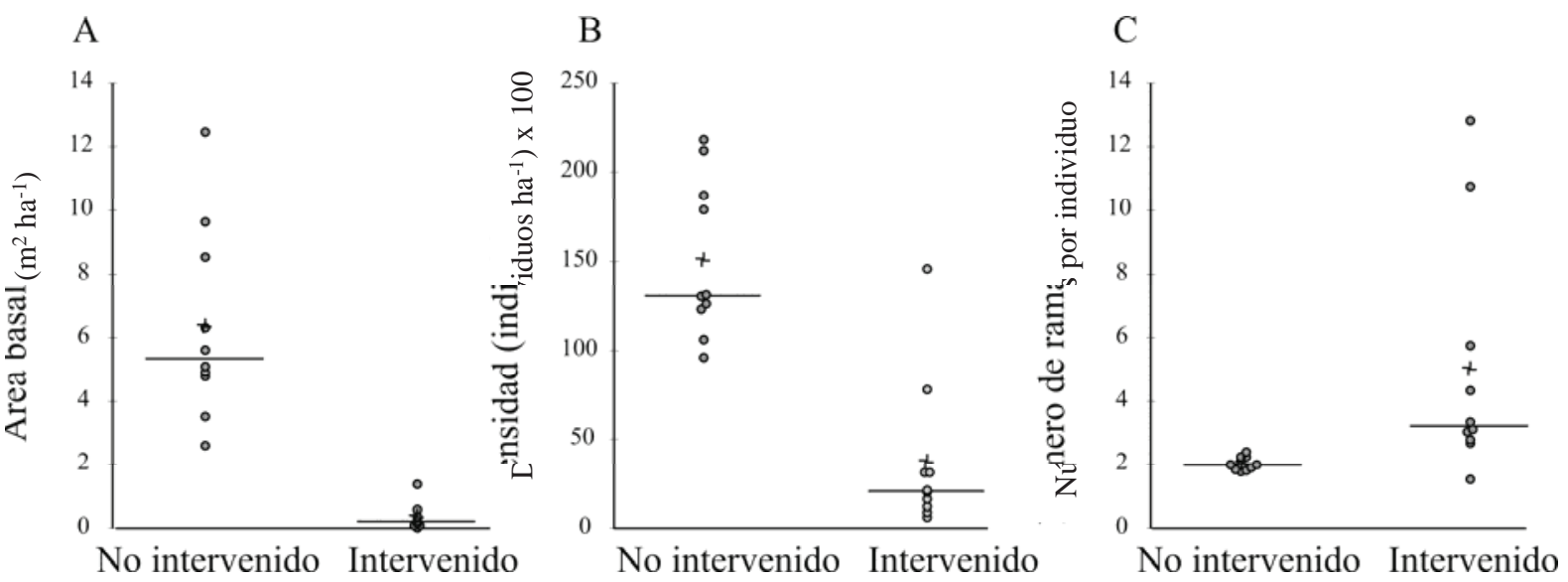

Figura 4. (A) Área basal, (B) densidad y (C) número de ramas promedio por individuo de plantas leñosas (con diámetro $>5$ mm) en ambos paleocauces con diferente intervención antrópica. Unidad de conservación Copo, Santiago del Estero, Argentina. La línea horizontal indica la mediana y la cruz la media aritmética.

(A) Basal area, (B) density and (C) average number of branches per individual of woody plants (with diameter $>5 \mathrm{~mm}$ ) in the two ancient watercourses with different human interventions, Copo Conservation Unit, Santiago del Estero, Argentina. Horizontal line: median; cross: arithmetic mean. 


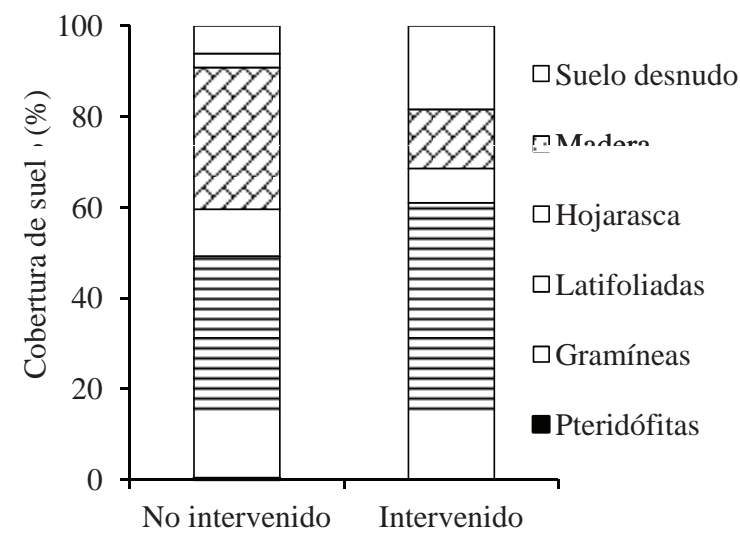

Figura 5. Cobertura del suelo de ambos paleocauces con diferente intervención antrópica. Unidad de Conservación Copo, Santiago del Estero, Argentina.

Soil cover in the two ancient watercourses with different human interventions, Copo Conservation Unit, Santiago del Estero, Argentina.

ciones, porque tenemos un solo pastizal independiente de cada situación. Para determinar el efecto de ambas perturbaciones sobre la comunidad de plantas leñosas sería necesario plantear un estudio a gran escala, con replicación verdadera de paleocauces (sin y con intervención antrópica), o bien un trabajo experimental en donde se apliquen de manera controlada los tratamientos relacionados con el régimen de fuego y el pastoreo por ganado. Sin embargo, dado que en este estudio los paleocauces comparados se desarrollan sobre una estructura geomorfológica similar, es posible que gran parte de lo encontrado esté explicado por las diferencias en la intervención humana (ganadería y fuego recurrente cada dos años aproximadamente) sobre ambas comunidades vegetales. Se debe tener en cuenta que al haber utilizado un diseño de niveles segregados en el espacio tendremos efectos alineados del fuego y el ganado, por lo que las diferencias encontradas en los pastizales podrían deberse a la acción conjunta del fuego y el ganado siendo imposible interpretar los efectos de cada actividad por separado. Aunque este hecho puede ser visto como una desventaja, se debe tener en cuenta que en el Chaco semiárido de Argentina ambas actividades (pastoreo de ganado y uso del fuego) son parte de la actividad productiva y se realizan en conjunto, por lo que los resultados son más cercanos a la realidad del ambiente local estudiado.

Distintos tipos de perturbaciones pueden afectar de manera diversa a las comunidades vegetales. Hay ejemplos en los que aumentan la riqueza del sistema en estudio (Sala et al. 1986, Collins et al. 1995, Kunst et al. 2003, Tálamo y Caziani 2003) y otros en que la disminuyen (Zedler et al. 1983, Tálamo y Caziani 2003). En este estudio, la riqueza específica total de plantas leñosas fue inferior en la zona con pastoreo de ganado exótico y un régimen de fuego recurrente en comparación con la riqueza total del paleocauce sin intervención antrópica. Pero por otro lado, comparando la riqueza de especies mediante el análisis de rarefacción, se encontró que la riqueza fue similar entre ambos pastizales, suponiendo que el pastizal sin intervención tuviera una abundancia de individuos similar a la del pastizal con intervenciones. Sin embargo, este supuesto carece de significado biológico para el sistema estudiado dado que los pastizales sin perturbaciones frecuentes presentan siempre una abundancia de plantas leñosas muy superior a la de los pastizales sometidos a fuegos recurrentes ${ }^{1}$. Por lo tanto, los resultados de este trabajo sugieren que las perturbaciones (ganadería y fuego recurrente) ocurridas en el pastizal con intervención podrían estar disminuyendo la riqueza específica de plantas leñosas del sistema. De manera similar, en otros pastizales mediterráneos se ha encontrado que la colonización de arbustos genera mayores valores de diversidad de plantas leñosas (Maestre et al. 2009). De todos modos, el ensamble de plantas leñosas es sólo un elemento del sistema estudiado, y también es importante tener en cuenta la posible relación de estas perturbaciones con otras plantas que presenten otras formas de vida. En este sentido, se ha encontrado que para un ambiente similar, los fuegos recurrentes son necesarios para mantener la riqueza y diversidad de plantas herbáceas (Kunst et al. 2003), por lo que la valoración de los efectos dependerá del grupo focal de interés y de los objetivos de manejo del área particular.

Según los modelos de colonización de leñosas en pastizales, los efectos del régimen de fuego y el pastoreo tendrían consecuencias opuestas en el proceso de colonización. Se podría predecir que en ambientes con alta frecuencia de incendios la colonización se impide o retrasa y por lo tanto existirá una dominancia marcada de plantas herbáceas, principalmente gramíneas. Por otro lado, si la carga de ganado es excesiva y el sistema está sobrepastoreado, se esperaría que la biomasa fina (gramíneas) disminuya notablemente dando lugar a un avance de la colonización de leñosas (Cabral et al. 2003, Briggs et al. 2005, Van Auken 2009). Dado que el paleocauce intervenido está afectado por ambas perturbaciones, y teniendo en cuenta que la fisonomía de este paleocauce correspondió a un pastizal dominado por aibe (Elionurus muticus) con baja presencia de plantas leñosas, proponemos que el régimen de fuego fue un factor mucho más importante que el pastoreo a la hora de determinar el resultado del proceso de colonización de plantas leñosas en este ambiente.

Del mismo modo, si bien no es posible diferenciar los efectos de la ganadería de los del régimen de fuego, éste último sería posiblemente un factor determinante de la alteración de la forma de crecimiento de los individuos leñosos debido a que el calor del fuego afecta a los tallos de las plantas, deshidratando los tejidos o matando yemas primarias y secundarias y disminuyendo la tasa de crecimiento. Esto podría estar ocurriendo en el paleocauce con intervención antrópica, en donde los individuos leñosos, además de ser de menor tamaño, presentaron un mayor

\footnotetext{
${ }^{1}$ Andrés Tálamo, observación personal.
} 
número de ramas que los individuos del paleocauce no intervenido ${ }^{2}$. En otros ambientes también se han reportado cambios en la arquitectura de las plantas en respuesta al fuego (Ginochio et al. 1994) y en respuesta a la herbivoría (Cooper et al. 2003). El fuego de pastizales, según su frecuencia e intensidad puede remover nuevamente la biomasa leñosa, eliminar localmente especies de arbustos y cambiar drásticamente las abundancias relativas de las diferentes especies de arbustos (Zedler et al. 1983, Collins et al. 1995).

En el presente estudio, tanto el régimen de fuego como el pastoreo del ganado podrían haber sido las causas de las diferencias encontradas en la cobertura del suelo en ambos pastizales. En la cubierta vegetal del pastizal sin intervención antrópica hubo mayor presencia de latifoliadas, hojarasca y madera al haber mayor densidad de árboles, arbustos y hierbas que al no ser consumidas por el ganado ni sufrir los efectos del fuego caen al suelo formando una capa protectora frente a la erosión hídrica y eólica, y generando potenciales micrositios para la regeneración, mientras que en el pastizal con perturbaciones antrópicas, debido a lo explicado anteriormente, hubo mayor presencia de gramíneas y suelo desnudo y no hubo presencia de madera.

El efecto de la ganadería y del régimen de fuego sobre la conservación de la biodiversidad y de los suelos es difícil de evaluar debido a la falta de relictos sin ganado y fuego, a la heterogeneidad del paisaje y a la complejidad de las respuestas (Kunst et al. 2003, Cingolani et al. 2005, 2008). Teniendo en cuenta esta realidad y que resulta también poco factible plantear estudios experimentales (manipulativos) que respeten la escala e intensidad de las perturbaciones estudiadas, estudios como éste, aunque tengan sus limitaciones, resultan muy útiles a la hora de generar información sobre la historia natural de los paleocauces y de su posible relación con perturbaciones locales, base fundamental para el manejo y conservación de sus comunidades.

\section{CONCLUSIONES}

Los dos paleocauces estudiados resultaron muy diferentes en todas las variables comparadas. En el paleocauce con fuego recurrente y ganadería se encontró una menor riqueza específica, área basal y densidad de plantas leñosas, diferente cobertura del suelo, diversidad y composición de especies, e individuos con un mayor número de ramas promedio en comparación a lo encontrado en el pastizal sin intervención antrópica. Es muy posible que gran parte de lo encontrado esté explicado por las diferencias en la intervención humana sobre ambos pastizales (ganadería y fuego) y se propone que el régimen de fuego sería un factor más importante que el pastoreo del ganado a la hora de definir el estado actual de las comunidades estudiadas. Para finalmente poder determinar el posible efecto del fuego y del pastoreo por ganado vacuno sobre el ensamble de

\footnotetext{
${ }^{2}$ Andrés Tálamo, observación personal.
}

plantas leñosas en pastizales naturales del Chaco, se recomienda hacer un estudio manipulativo trabajando con pobladores locales en la zona de la Reserva Provincial Copo, manejando la carga ganadera y la frecuencia del fuego, y evaluando la respuesta del sistema local bajo estudio.

\section{AGRADECIMIENTOS}

Agradecemos a la Administración de Parques Nacionales de Argentina por el financiamiento brindado que permitió la concreción del Programa de Monitoreo de la Biodiversidad del Área de Conservación Copo, dentro del cual se enmarca este trabajo. Especialmente agradecemos a quienes colaboraron con la recolección de datos en el campo (Carlos Trucco, Gustavo Maras, Yanina Bonduri y a los guardaparques del Parque Nacional Copo, Daniela Salazar, Mario Giménez y Mario González). Dos revisores anónimos contribuyeron a mejorar la calidad y claridad de este artículo.

\section{REFERENCIAS}

Bravo S, C Kunst, A Giménez, G Moglia. 2001. Fire regime of a Elionorus muticus Spreng. savanna, western Chaco region, Argentina. International Journal of Wildland Fire 10: 65-72.

Briggs JM, AK Knapp, JM Blair, JL Heisler, GA Hoch, MS Lett, JK McCarron. 2005. An ecosystem in transition: causes and consequences of the conversion of mesic grassland to shrubland. Bioscience 55: 243-254.

Cabral AC, JM De Miguel, AJ Rescia, MF Schmitz, FD Pineda. 2003. Shrub encroachment in Argentinean savannas. Journal of Vegetation Science 14: 145-152.

Caziani S, C Trucco, P Perovic, A Tálamo, E Derlindati, J Adámoli, L Lobo, M Fabrezi, M Srur, V Quiroga, M Martínez Oliver. 2003. Línea de Base y Programa de monitoreo de biodiversidad del Parque Nacional Copo. Informe final. Administración de Parques Nacionales, Argentina. 236 p.

Cingolani AM, I Noy-Meir, S Díaz. 2005. Grazing effects on rangeland diversity: a synthesis of contemporary models. Ecological Applications 15: 757-773.

Cingolani AM, I Noy-Meir, DD Reninson, M Cabido. 2008. La ganadería extensiva, ¿es compatible con la conservación de la biodiversidad y de los suelos? Ecología Austral 18: 253-271

Collins SL, SM Glenn, DJ Gibson. 1995. Experimental analysis of intermediate disturbance and initial floristic composition: decoupling cause and effect. Ecology 76: 486-492.

Cooper SM, MK Owens, DE Spalinger, TF Ginnett. 2003. The architecture of shrubs after defoliation and the subsequent feeding behavior of browsers. Oikos 100: 387-393.

Feinsinger P. 2004. El Diseño de Estudios de Campo para la Conservación de la Biodiversidad. Santa Cruz de la Sierra, Bolivia. Editorial FAN. 243 p.

Ginochio R, M Holmgren, G Montenegro. 1994. Effect of fire on plant architecture in Chilean shrubs. Revista Chilena de Historia Natural 67: 177-182.

Gotelli NJ, G Entsminger. 2002. Null models software for Ecology, version 7. Acquired Intelligence Inc. \& Kesey-Bear, Burlington, VT 05465, USA. Disponible en http://homepa- 
ges.together.net/ gentsmin/ecosim.htm.

Kunst C, S Bravo, J Panigatti. 2003. Fuego en los Ecosistemas Argentinos. Santiago del Estero, Argentina. INTA. 332 p.

Kunst C, S Bravo, F Moscovich, J Herrera, J Godoy, S Vélez. 2003. Fecha de aplicación de fuego y diversidad de herbáceas en una sabana de Elionorus muticus (Spreng) O. Kuntze. Revista Chilena de Historia Natural 76: 105-115.

Maestre NT, MA Bowker, MD Puche, MB Hinojosa, I Martínez, P García-Palacios, AP Castillo, S Soliveres, AL Luzuriaga, AM Sánchez, JA Carreira, A Gallardo, A Escudero. 2009. Shrub encroachment can reverse desertification in semi-arid Mediterranean grasslands. Ecology Letters 12: 930-941.

McCune B, JB Grace, DL Urban. 2002. Analysis of ecological communities. MjM Software Design. Oregon, USA. 300 p.

McCune B, M Mefford. 1999. PC-ORD. Multivariate Analysis of Ecological Data, Version 4 for Windows. MjM Software Design, Gleneden Beach, Oregon, USA.

Millennium Ecosystem Assessment 2005. Ecosystems and Human Well-being: Desertification Synthesis. World Resources Institute, Washington D.C. 26 p.

Morello J. 1970. Modelo de relaciones entre pastizales y leñosas colonizadoras en el Chaco argentino. IDIA 276: 31-52.

Morello J, C Saravia Toledo. 1959 a. El bosque chaqueño I. Paisaje primitivo, paisaje natural y paisaje cultural en el oriente de Salta. Revista Agronómica del Noroeste Argentino 3: 5-81.

Morello J, C Saravia Toledo. 1959 b. El bosque chaqueño II. La ganadería y el bosque en el oriente de Salta. Revista Agronómica del Noroeste Argentino 3: 209-258.

Morello J, J Adámoli. 1968. Las grandes unidades de vegetación y ambientes del Chaco Argentino. I Parte: objetivos y metodología. Revista de Investigaciones Agropecuarias 10: 1-125.

Neumann R. 1999. Ecología y Manejo del Chaco semiárido. Sal- ta, Argentina. INTA- EEA. 6 p.

Perovic P, C Trucco, A Tálamo, D Ramallo, A Baumgartner, A Lacci, F Mohr, Y Bonduri, V Quiroga. 2008. Primer monitoreo de la biodiversidad de la Unidad de Conservación Copo. Informe Final. Programa de Monitoreo de la Biodiversidad del Parque Nacional Copo, Reserva Provincial Copo y zona de Amortiguamiento. APN/GEF/BIRF. 77 p.

Prado D. 1993. What is the Gran Chaco vegetation in South America? I. A review. Contribution to the study of flora and vegetation of the Chaco. V. Candollea 48(1): 145-172.

Sala OE, M Oesterheld, C León, A Soriano. 1986. Grazing effects upon plant community structure in subhumid grasslands of Argentina. Vegetatio 67: 27-32.

Tálamo A, S Caziani. 2003. Variation in woody vegetation among sites with different disturbance histories in the Argentine Chaco. Forest Ecology and Management 184: 79-92.

Tálamo A. 2006. Biodiversidad de plantas leñosas y disturbios humanos en el bosque chaqueño semiárido: efectos del aprovechamiento forestal. Tesis Doctoral. Buenos Aires, Argentina. Facultad de Cs. Exactas y Naturales, Universidad de Buenos Aires. 110 p.

Van Auken OW. 2000. Shrub invasions of North American semiarid grasslands. Annual Review of Ecology and Systematics 31: 197-215.

Van Auken OW. 2009. Causes and consequences of woody plant encroachment into western North American grasslands. Journal of Environmental Management 90(10): 2931-2942.

Zar J. 1999. Biostatical analysis. Fourth Edition. New Jersey, USA. Prentice-Hall, Inc. 663 p.

Zedler PH, CR Gautier, GS McMaster. 1983. Vegetation changes response to extreme events: the effect of the short interval between fires in California chaparral and coastal scrub. Ecology 64: 809-818. 